\title{
Autodoação e autotransfusão de sangue pré-doado em cirurgia cardíaca com circulação extracorpórea
}

Mário Gesteira COSTA*, Frederico VASCONCELOS*, Roberto PEREIRA*, Carlos Antônio SILVEIRA*, Esdras GASPAR*, Gilberto GODOY*, Milton GARRET*, Maria de Lourdes SOUZA*, Antônio Carlos LEÃO*

COSTA, M. G.; VASCONCELOS, F.; PEREIRA, R.; SILVEIRA, C. A.; GASPAR, E.; GODOY, G.; GARRET, M.; SOUZA, M. L.; LEÃO, A. C. - Autodoação e autotransfusão de sangue pré-doado em cirurgia cardiaca com circulação extracorpórea. Rev. Bras. Cir. Cardiovasc., 4(2): 143-150, 1989.

RESUMO: Estudo prospectivo, entre agosto de 1987 e setembro de 1988, em 80 pacientes adultos submetidos a cirurgia cardiaca eletiva com circulação extracorpórea (CEC) aferindo a eficácia da autodoação $(A D)$ e autotransfusāo (AT) em reduzir o uso de sangue e hemoderivados homólogos (SDH). O Grupo Controle $(G C)$ não foi submetido a $A D(n=38)$. Coleta pré-operatória de sangue foi realizada em 42 pacientes, constituindo o Grupo Autodoação (GAD), iniciando-se de um a sete dias pré-operatórios (GAD I $n=29$ ) e entre oito a 14 dias pré-operatórios (GAD ॥ $n=13$ ). Os Grupos $G C$ e GAD foram bastante semelhantes nos seus parâmetros pré, intra e pós-operatórios. Os resultados demonstraram que a utilização de hemoderivados foi similar, nos diversos grupos. Contudo, o número de pacientes expostos a SDH: GC $27(71 \%)$ $x$ GAS $10(23,8 \%), p<(0,001)$; o volume médio de SHD (GC $1241 \mathrm{ml}$ x GAD $412 \mathrm{ml}, \mathrm{p}<0,003)$ e o número médio de unidades homólogas utilizadas (GC 6,31 x GAD 1,95, $p<0,001$ ) demonstraram que $A D$ e $A T$ foram eficazes em reuzir em $64 \%$ o volume médio de SDH, diminuindo a exposição a unidades homólogas e minimizando o número de pacientes expostos. O GAD II obteve os melhores índices, mas não atinge significado estatístico quando comparado ao GAD I, talvez devido ao pequeno número de pacientes. Acreditamos que AD e AT devem ser incentivadas em cirurgia cardiaca eletiva. corpórea.

DESCRITORES: sangue, autodoação; sangue, autotransfusão; sangue, conservação; circulaçāo extra-

\section{INTRODUÇÃO}

O número crescente de cirurgias cardiacas tem contribuido para o consumo das reservas dos bancos de sangue $^{15.21}$ e o consumo de sangue e hemoderivados homólogos $(\mathrm{SDH})$ é associado a uma morbidade bem conhecida, podendo, até, ser letal ${ }^{1}, 2,12,14,22$.

A introdução de hemodiluição ${ }^{26}$, o desenvolvimento de sistemas de centrifugação e filtragem do sangue perdido em aspiradores externos ${ }^{24}$, a reinfusāo do volume residual do exigenador ${ }^{19}$, a autotransfusão de sangue coletado no intra-operatório ${ }^{12}$, a autotransfusão de san- gue autógeno pré-doado ${ }^{27}$, a autotransfusāo do sangue drenado do mediastino no pós-operatório ${ }^{13}$ e a aceitação de anemia normovolêmica assintomática têm resultado em uma diminuição do consumo de $\mathrm{SDH}$, em cirurgia cardiaca com circulaçāo extracorpórea; nos últimos 13 anos, o consumo decresceu de oito unidades em $1973^{24}$, para uma a três unidades em $1986^{3-5,13}, 16.23 .25$.

Embora a autodoaçāo (AD) e autotransfusão (AT) de sangue pré-doado tenham sido eficazes em reduzir o uso de $\mathrm{SDH}^{6,7}, 16 \cdot 18,20$, o meio médico tem relutado em aceitar estas técnicas, nāo sendo rotina, na maioria dos Serviços. 
COSTA, M. G.; VASCONCELOS, F.; PEREIRA, R.; SILVEIRA, C. A.; GASPAR, E.; GOdOY, G.; GARRET, M.; SOUZA, M. L.; LEẢO, A. C. - Autodoação e autotransfusão de sangue pré-doado em cirurgia cardíaca com circulação extracorpórea. Rev. Bras. Cir. Cardiovasc., 4(2): 143-150, 1989.

Neste estudo prospectivo, relatamos nossa experiência com $A D$ e $A T$ e analisamos a eficácia destas técnicas em diminuir o uso de SDH.

\section{CASUISTICA E MÉTODOS}

Entre agosto de 1987 e setembro de 1988, todos os pacientes avaliados para cirurgia cardiaca com circulação extracorpórea (CEC), em nosso Serviço, foram submetidos a um protocolo de inclusāo neste estudo prospectivo (Tabela 1); 31 pacientes foram excluidos do estudo, por não preencherem os critérios, sendo 23 por idade abaixo dos 20 anos, cinco necessitaram de cirurgia urgente, dois por apresentarem lesão de tronco de coronária esquerda e um por coagulopatia pré-operatória.

Foi oferecida, aos 80 pacientes aceitos para estudo, a opção de autodoação e autotransfusão, ou tratamento convencional. Trinta e oito optaram pelo tratamento convencional e formaram o Grupo Controle $(G C n=38)$. Os 42 pacientes que optaram pela autodoação e autotransfusāo formaram o Grupo GAD ( $n=42)$, sendo iniciados em sulfato ferroso $300 \mathrm{mg}$ três vezes por dia e dieta rica em ferro, tendo seu sangue coletado entre um e 14 dias pré-operatórios.

As coletas foram realizadas na unidade de terapia intensiva (UTI), sendo os pacientes assistidos por um cardiologista e um hemoterapeuta e monitorizados em seus parâmetros clínicos, eletrocardiograma contínuo, eletrocardiograma pré e pós-autodoaçāo e parâmetros hematimétricos.

O sangue foi conservado em ácido citrato dextrose (ACD) e estocado em banco de sangue, sob a forma de sangue total, ou fracionado em plasma fresco congelado e concentrado de hemácias. Em dois pacientes, foi realizada apenas a coleta intra-operatória de $500 \mathrm{ml}$ de sangue, retirado do átrio direito após a indução anestésica, antes da heparinização, e conservados em ACD. Outros 10 pacientes tiveram, além da autodoação pré-operatória, a coleta de $500 \mathrm{ml}$ de sangue intra-operatória, como descrito acima.

TABELA 1

\section{CRITÉRIOS DE INCLUSĀO}

Peso corporal acima de $50 \mathrm{~K}$
Idade acima de 20 anos
Hematócrito $36 \%$
Cirurgia eletiva
Ausência de:
- anemia
- hipoproteinemia
— infecção
- lesão de tronco de coronária esquerda
- distúrbios de coagulação
- angina instável na fase de instabilidade

Os dois grupos tiveram parâmetros bastante semeIhantes, quanto a idade sexo, hematócrito pré-operatório, hematócrito de CEC, classe funcional, duraçāo da anóxia e da CEC (Tabela 2), assim como quanto aos procedimentos cirúrgicos realizados (Tabela 3 ), ou parâmetos pós-operatórios, incluindo hematócrito de chegada à UTI, perda sangüínea pós-operatória, hematócrito de alta, reoperação por sangramento e número de óbitos ( $\mathrm{Ta}$ bela 4).

Todos os pacientes foram operados com CEC, utilizando-se um primming de Ringer lactato e glicose a $5 \%$ sem hemoderivados, oxigenadores de bolhas e hipotermia a $26^{\circ} \mathrm{C}$. A proteção miocárdica foi obtida com cardioplegia hipercalêmica hipotérmica e hipotermia tópica.

Ao fim da CEC, o volume residual do exigenador foi repassado aos pacientes, ou seqüestrado no reservatório de cardiotomia e, subseqüentemente, transfundido. Não foram utilizados sistemas de centrifugação e filtragem do sague perdido em aspiradores externos, nem para processamento do sangue residual do oxigenador. Também năo foi utilizada a reinfusão do sangue coletado do mediastino, no pós-operatório. O Grupo GC teve suas necessidades transfusionais supridas por SDH; o Grupo GAD recebeu, preferencialmente, sangue autógeno.

As necessidades transfunsionais de cada paciente, incluindo sangue e hemoderivados, foi computada considerando os períodos intra, pós e per-operatório. A análise estatística foi realizada, utilizando o teste de Qui-quadrado, o teste $\mathrm{T}$ de Student e a prova nâo paramétrica de Mann-Whitney.

Para efeito de análise, o Grupo GAD foi, retrospectivamente, subdividido em GAD I, incluindo os pacientes cuja autodoação foi de $500 \mathrm{ml}$, e iniciada entre um e sete dias pré-operatórios ( $n=29$ ) e GAD II: com duas ou mais coletas, iniciando entre oito e 14 pré-operatórios ( $n=13$ ). Dois pacientes, cujo sangue foi coletado apenas no intra-operatório, foram incluídos no Grupo GAD I.

\section{RESULTADOS}

Foram realizadas 52 coletas pré-operatórias e 12 intra-operatórias, totalizando 64 unidades autógenas (média 1,52 unidades/paciente); excluindo as coletas intra-operatórias, foram obtidas, em mdia, 1,23 unidades autógenas por paciente.

O Grupo GAD teve sua autodoação realizada, em média, 6,3 dias antes da operação; este intervalo foi similar no Grupo GAD I (5,8 dias). O Grupo GAD II teve sua coleta inicial, em média, 10,6 dias antes da operaçāo, seguida de uma segunda coleta 5,7 dias antes do ato cirúrgico. 
COSTA, M. G.; VASCONCELOS, F.; PEREIRA, R.; SILVEIRA, C. A.; GASPAR, E.; GOdOY, G.; GARRET, M.; SOUZA, M. L.; LEÃO, A. C. - Autodoação e autotransfusão de sangue pré-doado em cirurgia cardíaca com circulação extracorpórea. Rev. Bras. Cir. Cardiovasc., 4(2): 143-150, 1989.

TABELA 2

CARACTERISTICAS DA POPULAÇĀO EM ESTUDO

\begin{tabular}{|c|c|c|c|c|}
\hline Variável & 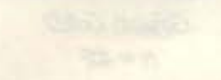 & $\begin{array}{c}\text { Grupo GC } \\
(n=38)\end{array}$ & $\begin{array}{c}\text { Grupo GAD } \\
(n=42)\end{array}$ & $p$ \\
\hline Idade (anos) & & $45,6 \pm 2,47$ & $40,7 \pm 2,01$ & $>0,02 \mathrm{NS}^{* *}$ \\
\hline Número/sexo & & $18 \mathrm{~m} / 20 \mathrm{f}$ & $20 \mathrm{ml} / 22 \mathrm{f}$ & $>0,05 \mathrm{NS}^{* * *}$ \\
\hline Hematócrito pré-op" & & $41,9 \pm 0,84$ & $39,3 \pm 0,46$ & $<0,05 \quad \cdots$ \\
\hline NYHA I e II/III e IV & & $10 / 28$ & $05 / 37$ & $>0,01 \mathrm{NS}^{* * *}$ \\
\hline Tempo de anóxia (min) $)^{*}$ & & $45,9 \pm 3,86$ & $57,2 \pm 5,05$ & $>0,05 \mathrm{NS}^{* *}$ \\
\hline Tempo de CEC (min)* & & $90,1 \pm 5,85$ & $101,6 \pm 7,88$ & $>0,05 \mathrm{NS}^{* *}$ \\
\hline Hematócrito de CEC* & & $24,1 \pm 0,62$ & $22,1 \pm 0,67$ & $<0,05 \quad \cdots$ \\
\hline
\end{tabular}

- Média e standard erro da média; "* teste T de Student; ${ }^{* *}$ Qui quadrado; NYHA = classe funcional; NS = não significante; CEC = circulaçāo extracorpórea.

TABELA 3

PROCEDIMENTOS CIRÚRGICOS

\begin{tabular}{lcc}
\hline Operação & Grupo GC & Grupo GAD \\
\hline Revascularizaçăo miocárdica & 16 & 15 \\
Comissurotomia mitral & 06 & 07 \\
Prótese mitral & 07 & 07 \\
Prótese aórtica & 04 & 01 \\
Mitro-aórticos & 03 & 07 \\
C.I.A. & 02 & 03 \\
Aneurisma aorto ascendente & - & 01 \\
Revasc. miocárdica e prótese & - & 01 \\
\hline
\end{tabular}

C.I.A. = comunicação interatrial; ${ }^{*}$ análise por Qui-quadrado entre grupos, por operaçăo, demonstra $p>0,05$ (não significante).

O hematócrito "pré-autodoaçăo" foi $41,7 \pm 0,64 \%$ e, comparado ao hematócrito "pré-operatório" do Grupo GC $(\bar{X}=41,9 \pm 0,84 \%)$, demonstrou não haver diferença significante ( $p>0,05$ NS-teste T de Student). No entanto, a autodoação causou uma queda média de 2,4 pontos percentuais, entre hematócrito pré-autodoação e pré-operatório do Grupo GAD, resultando em menor hematócrito pré-operatório e durante CEC, quando comparado ao GC ( $p<0,05)$.

Apenas um paciente $(2,4 \%)$ apresentou complicação da autodoação, conssitindo em um episódio de hipo- tensāo leve e transitória, sem apresentar sintomas ou alteraçōes eletrocardiográficas significantes e revertendo, rapidamente, com hidratação intravenosa com solução cristalóide.

A Tabela 5 mostra o uso de hemoderivados, em volume, nas diversas fases do tratamento. A necessidade global transfusional, em cada fase, foi similar nos dois grupos; as difereças não atingem significado estatístico e isto reflete os critérios transfusionais similares nos dois grupos. A utilização de SDH foi distintamente menor no Grupo GAD, em todas as fases, alcançando significado estatístico. As unidades autógenas supriram $81 \%$ do volume utilizado no intra-operatório e $49 \%$ no pós-operatório do Grupo $G A D$, resultando que $64 \%$ do volume utilizado no período per-operatório foi proveniente de unidades autoǵenas.

A Tabela 6 demonstra o número de pacientes expostos a hemoderivados, em cada grupo. No Grupo GC, 17 pacientes $(44,7 \%)$ necessitaram de hemoderivados no intra-operatório e 19 pacientes $(50 \%)$ no pós-operatório; considerando o período per-operatório, $71 \%$ dos pacientes necessitaram de SDH.

O maior percentual demonstrado, para o Grupo GAD, de pacientes expostos a hemoderivados (qualquer que seja a origem), tanto no intra-operatório $(64,3 \%)$ como no pós-operatório $(69 \%)$, não atinge significado

TABELA 4

CARACTERISTICAS PÓS-OPERATÓRIAS

\begin{tabular}{|c|c|c|c|}
\hline Variável & Grupo GC & Grupo GAD & $\rho$ \\
\hline Hematócrito UTI §" & $32,1 \pm 0,70$ & $31,9 \pm 0,69$ & $>0,05$ NS $* *$ \\
\hline Perda sangüínea $(\mathrm{ml})^{*}$ & $679 \pm 106$ & \pm 91 & $>0,05$ NS $^{* *}$ \\
\hline Hematócrito de alta* & $31,1 \pm 0,87$ & $31,0 \pm 0,78$ & $>0,05 \mathrm{NS}^{* *}$ \\
\hline Reop. por hemorragia & $02(5,2 \%)$ & $02(4,7 \%)$ & $>0,05 \mathrm{NS}^{* *}$ \\
\hline Óbitos & $03(7,9 \%)$ & $03(7,1 \%)$ & $>0,05 \mathrm{NS}^{* . *}$ \\
\hline
\end{tabular}

$\S^{*}$ " hematócrito de chegada à unidade de terapia intensiva; " média e standard erro da média; “" teste T de Student; $\cdots$ Qui quadrado; Reop. = reoperação. 
COSTA, M. G.; VASCONCElOS, F.; PEREIRA, R.; SILVEIRA, C. A.; GASPAR, E.; GodoY, G.; GARRET, M.; SOUZA, M. L.; LEĀO, A. C. - Autodoação e autotransfusāo de sangue pré-doado em cirurgia cardiaca com circulação extracorpórea. Rev. Bras. Cir. Cardiovasc., 4(2): 143-150, 1989.

TABELA 5

UTILIZAÇĀO DE HEMODERIVADOS.

\begin{tabular}{|c|c|c|c|}
\hline 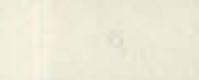 & $\begin{array}{c}\text { Grupo GC } \\
n=38\end{array}$ & $\begin{array}{c}\text { Grupo } G A D \\
n=42\end{array}$ & $p$ \\
\hline \multicolumn{4}{|l|}{ INTRA-OP. } \\
\hline SDH & $448 \pm 129$ & $98,8 \pm 43,5$ & $p<0,004^{* *}$ \\
\hline SDA & $448 \pm 129$ & $430,9 \pm 62,0$ & \\
\hline TOTAL & $448 \pm 129$ & $529,7 \pm 77,9$ & $p>0,05$ NS $^{* *}$ \\
\hline \multicolumn{4}{|l|}{ POS-OP. } \\
\hline $\begin{array}{l}\text { SDH } \\
\text { SDA }\end{array}$ & $774,8 \pm 161,9$ & $\begin{array}{r}308,3 \pm 118,6 \\
297,6 \pm 47,9\end{array}$ & $p<0,01^{* *}$ \\
\hline TOTAL & $774,8 \pm 161,9$ & $605,9 \pm 119,9$ & $p>0,04$ NS $^{* *}$ \\
\hline \multicolumn{4}{|l|}{ PER-OP. } \\
\hline SDH & $1222,8 \pm 239,7$ & $407,1 \pm 134,6$ & $p<0,003^{* *}$ \\
\hline SDA & & $728,5 \pm 47$ & \\
\hline TOTAL & $1222,8 \pm 239,7$ & $1135,6 \pm 137,6$ & $p>0,2$ NS $^{*}$ \\
\hline
\end{tabular}

TABELA 6

PACIENTES EXPOSTOS A HEMODERIVADOS

\begin{tabular}{|c|c|c|c|}
\hline & Grupo GC & Grupo GAD & $p$ \\
\hline \multicolumn{4}{|l|}{ INTRA-OP. } \\
\hline $\begin{array}{l}\text { SHD } \\
\text { SDA }\end{array}$ & $17(44,7 \%)$ & $\begin{array}{l}05(11,9 \%) \\
27(64,3 \%)\end{array}$ & $<0,01^{*}$ \\
\hline $\begin{array}{l}\text { TOTAL } \\
\text { PÓS-OP. }\end{array}$ & $17(44,7 \%)$ & $27(64,3 \%)$ & $>0,1 \mathrm{NS}^{*}$ \\
\hline SDH & $19(50 \%)$ & $08(19 \%)$ & $<0,01^{\circ}$ \\
\hline SDA & & $29(69 \%)$ & \\
\hline TOTAL & $19(50 \%)$ & $29(69 \%)$ & $>0,1 \mathrm{NS}^{*}$ \\
\hline PER-OP. & & & \\
\hline $\begin{array}{l}\text { SDH } \\
\text { SDA }\end{array}$ & $27(71 \%)$ & $\begin{array}{ll}10 & (23,8 \%) \\
41 & (97,6 \%)\end{array}$ & $<0,001^{\circ}$ \\
\hline TOTAL & 27 (71\%) & $41(97,6 \%)$ & $<0,01^{*}$ \\
\hline
\end{tabular}

SDA = sangue e hemoderivados autógenos; $\mathrm{SDH}=$ sangue e hemoderivados homólgos; * Qui quadrado; NS = não significante.

estatístico quando comparado ao $\mathrm{GC}$, nas fases respectivas. Esta exposição, no entanto, foi, sem dúvida, maior no Grupo GAD, send isto comprovado pela análise de periodo per-operatório, onde $97,6 \%$ dos pacientes do GAD utilizaram hemoderivados ( $p<0,01$ ), refletindo maior liberalidade em iniciar transfusão autógena.

O número de pacientes expostos a SDH foi nítidamente menor no Grupo GAD, em todas as fases, e alcançando, em todas, significado estatístico. No per-operatório, apenas 10 pacientes $(23,8 \%)$ do Grupo GAD, contra 27 pacientes $(71 \%)$ do Grupo GC, necessitaram sangue e hemoderivados homólogos $(p<0,001)$.

O número médio de unidades homólogas utilizadas por cada grupo reflete o número de doadores a que os pacientes foram expostos (Tabela 7). Em todas as fases, a diferença demonstrando menor exposição do
TABELA 7 UNIDADES DE HEMODERIVADOHOMOLOGOUTILIZADAS*

\begin{tabular}{cccc}
\hline & Grupo GC & Grupo GAD & $p$ \\
\hline INTRA-OP. & & & \\
C/PLAQ. & $3,26 \pm 0,95$ & $0,69 \pm 0,38<0,02^{* *}$ \\
S/PLAQ. & $1,03 \pm 0,30$ & $0,21 \pm 0,09<0,02^{* *}$ \\
POS-OP. & & & \\
C/PLAQ. & $3,05 \pm 0,76$ & $1,26 \pm 0,64<0,01^{* *}$ \\
S/PLAQ. & $2,18 \pm 0,45$ & $0,78 \pm 0,29<0,01^{* *}$ \\
PER-OP. & & & \\
C/PLAQ. & $6,31 \pm 1,29$ & $1,95 \pm 0,78<0,001^{* *}$ \\
S/PLAQ. & $3,21 \pm 0,62$ & $1,00 \pm 0,32<0,001^{* *}$ \\
\end{tabular}

$\mathrm{C} / \mathrm{PLAQ} .=$ incluindo plaquetas; $\mathrm{S} / \mathrm{PLAQ} .=$ excluindo plaquetas; " média e estandard erro da média; "* análise por MannWhitney.

Grupo GAD atinge significado estatístico. O Grupo GC utilizou uma média de 6,31 unidades homólogas/paciente, no per-operatório, contra 1,95 unidades homólogas/ paciente do Grupo GAD ( $p<0,001)$.

A análise dos subgrupos GAD I e GAD II, quanto à utilização de hemoderivados em volume (Tabela 8 ), quanto ao número de pacientes expostos a hemoderivados (Tabela 9) e quanto ao número médio de unidades homlogas utilizadas por paciente (Tabela 10), embora demonstre uma diferença em favor de uma menor utilização de SDH pelo GAD II, não atinge significado estatístico, provavelmente devido ao pequeno número de pacientes em estudo.

\section{COMENTÁRIOS}

A cirurgia cardíaca com CEC tem contribuído para o consumo dos estoques dos bancos de sangue. Em 
COSTA, M. G.; VASCONCELOS, F.; PEREIRA, R.; SILVEIRA, C. A.; GASPAR, E.; GOdOY, G.; GARRET, M.; SOUZA, M. L.; LEĀO, A. C. - Autodoação e autotransfusâo de sangue pré-doado em cirurgia cardiaca com circulação extracorpórea. Rev. Bras. Cir. Cardiovasc., 4(2): 143-150, 1989.

TABELA 8

UTILIZAÇĀO DE HEMODERIVADOS

\begin{tabular}{|c|c|c|}
\hline & Grupo GAD I & Grupo GAD // \\
\hline \multicolumn{3}{|l|}{ INTRA-OP. } \\
\hline SDH & $143,1 \pm 61,6$ & - \\
\hline SDA & $324,1 \pm 56,7$ & $669,2 \pm 137,4$ \\
\hline TOTAL & $467,2 \pm 94,0$ & $669,2 \pm 137,4>0,05 \mathrm{NS}^{* *}$ \\
\hline \multicolumn{3}{|c|}{ POS-OP. } \\
\hline SDH & $363,8 \pm 156,5$ & $184,6 \pm 161,3>0,3 \mathrm{NS}^{* *}$ \\
\hline SDA & $275,8 \pm 53,1$ & $346,1 \pm 101,6$ \\
\hline TOTAL & $639,6 \pm 159,5$ & $530,7 \pm 160,2>0,4 \mathrm{NS}^{* *}$ \\
\hline \multicolumn{3}{|l|}{ PER-OP } \\
\hline SDH & $506,9 \pm 179,6$ & $184,6 \pm 161,3>0,2 \mathrm{NS}^{* *}$ \\
\hline SDA & $600,0 \pm 46,0$ & $1015 \pm 58,6$ \\
\hline TOTAL & $1106,9 \pm 184,4$ & $1200,0 \pm 177,9<0,05^{* *}$ \\
\hline
\end{tabular}
de Mann Whitney; SDH = sangue e hemoderivados homologos; SDA = sangue e hemoderivados autógenos.

TABELA 9

PACIENTES EXPOSTOS A HEMODERIVADOS.

\begin{tabular}{|c|c|c|c|}
\hline & Grupo GAD I & Gaupo GAD // & $p$ \\
\hline \multicolumn{4}{|l|}{ INTRA-OP. } \\
\hline SDH & $05(17,2)$ & - & \\
\hline SDA & $17(58,6)$ & $10(76.9)$ & $>0,05 \mathrm{NS}^{* *}$ \\
\hline TOTAL & $17(58,6)$ & $10(76,9)$ & $>0,05 \mathrm{NS}^{* *}$ \\
\hline \multicolumn{4}{|l|}{ PÓS-OP. } \\
\hline $\mathrm{SDH}$ & $06(20,6)$ & $02(15,3)$ & $>0,05 \mathrm{NS}^{* *}$ \\
\hline SDA & $15(51,7)$ & $07(53,8)$ & $>0,05 \mathrm{NS}^{* *}$ \\
\hline TOTAL & $20(68,9)$ & $09(69,2)$ & $>0,2 \mathrm{NS}^{* *}$ \\
\hline \multicolumn{4}{|l|}{ PER-OP. } \\
\hline SDH & $08(27,5)$ & $02(15.3)$ & $>0.5$ NS $^{* *}$ \\
\hline SDA & $28(96,5)$ & $13(100)$ & \\
\hline TOTAL & $28(96,5)$ & $13(100)$ & \\
\hline
\end{tabular}

- número de pacientes expostos; números entre parênteses representam percentual; $\mathrm{SDH}=$ sangue e hemoderivado homólogo; SDA = sangue de hemoderivado autógeno; $\mathrm{NS}=$ não significante; "“ análise por Qui-quadrado.

1973, ROCHA \& STENGLE ${ }^{21}$ alertaram para a necessidade de sistematizar o uso de hemoderivados, considerando o crescimento progressivo da cirurgia cardíaca. Nos Estados Unidos da América, estima-se que $\mathbf{4 7 4 . 0 0 0}$ cirurgias cardiacas foram realizadas em 1985 e projeta-se que serāo realizadas 683.000 em 1995, num crescimento de $44 \%{ }^{15}$.

A morbidade da transfusão de sangue e hemoderivados homólogos é bem conhecida. As complicações de hemotransfusão, incluindo reaçōes hemoliticas agudas e tardias, insuficiência respiratória, contaminação bacteriana e hepatite, chegam, às vezes, ao êxito letal ${ }^{12}$. $\mathrm{A}$ hepatite não $\mathrm{A}$, não $\mathrm{B}$ é responsável por 60 a $90 \%$ das hepatites pós-transfusionais, ocorrendo em 6,7 a $11 \%$ dos pacientes submetidos a cirurgia cardiaca e tendo uma forte tendência à cronicidade. Há, também, o risco de contrair a síndrome de imuno-deficiência adqui-
TABELA 10 UNIDADES DE HEMODERIVADO HOMÓLOGO

\begin{tabular}{ccc}
\hline \multicolumn{4}{c}{ Grupo GAD / } & GrupO GAD II & $p$ \\
\hline INTRA-OP. \\
C/PLAQ. & $1,00 \pm 0,55$ & - \\
S/PLAQ. & $0,31 \pm 0,14$ & - \\
POS-OP. & & \\
C.PLAQ. & $1,58 \pm 0,90$ & $0,53 \pm 0,46>0,3$ NS** \\
S/PLAQ. & $0,89 \pm 0,38$ & $0,53 \pm 0,46>0,3$ NS** \\
PER-OP. & & \\
C/PLAQ. & $2,58 \pm 1,09$ & $0,53 \pm 0,46>0,2$ NS** \\
S/PLAQ. & $1,20 \pm 0,42$ & $0,53 \pm 0,46>0,2$ NS** \\
\end{tabular}

- número médio e standard erro da méria de unidades homólogas transfunidas; "* análise por teste de Mann Whitney; C/ $\mathrm{PLAQ}$. = incluindo plaquetas; $\mathrm{S} / \mathrm{PLAQ}$. = excluindo plaquetas.

rida, que se estima em quatro por cada 1.000 .000 de transfusōes ${ }^{1,2,14}$.

O número crescente de cirurgias cardíacas, aliado aos estoques escassos e à morbidade associada ao uso de SDH, levou ao desenvolvimento de técnicas de conservação de sangue, incluindo hemodiluição ${ }^{26}$, sistemas de centrifugação e filtragem do sangue perdido em aspiradores externos ${ }^{24}$, reinfusão do volume residual do oxigenador $^{19}$, autotranfusão de sangue coletado no intra-operatório ${ }^{11}$, autotransfusão de sangue pré-doado ${ }^{7}$, autotransfusão de sangue drenado do mediastino no pós-operatório ${ }^{13}$ e aceitação de anemia normovolêmica assintomática ${ }^{23}$. $\mathrm{O}$ esforço em conservar sangue resultou em uma diminuição do consumo de $\mathrm{SDH}$, vindo de oito unidades por paciente em 1973 (24), para uma a três unidades em $1986^{3-5,13,16,23,25}$.

Embora $A D$ e $A T$ resultem numa redução do uso de $\mathrm{SDH}^{6,7}$. 16-18. ${ }^{20}$, o meio médico tem relutado em aceitar estas técnicas. Concepçōes errôneas julgam dificil o estabelecimento da rotina, a aplicabilidade na maioria dos casos, a aceitação pelos pacientes, a capacidade do paciente em regenerar a volemia e células vermelhas, o risco em pacientes cardiacos, os custos e os benefícios alcançados.

A rotina establecida em nosso Serviço foi facilmente assimilada pelos médicos e pessoal para-médico, devido a interação entre o banco de sangue e o serviço de cirurgia cardíaca.

O fato de que $80(72,7 \%)$, dentre 110 pacientes operados no mesmo período, preencheram os requisitos para $A D$ e $A T$, sugere a aplicabilidade na maioria dos casos; considerando que neste estudo foram excluídos pacientes com idade abaixo dos 20 anos, nos quais a autodoação pode ser realizada com segurança ${ }^{18}$, este percentual deverá ser superior. Dentre os 80 paciente a quem foi oferecida a opção de AD e AT, 42 pacientes aceitaram (52,5\%); este percentual deverá subir mediante um trabalho de conscientização da população. Du- 
COSTA, M. G.; VASCONCELOS, F.; PEREIRA, R.; SILVEIRA, C. A.; GASPAR, E.; GOdOY, G.; GARRET, M.; SOUZA, M. L.; LEĀO, A. C. - Autodoação e autotransfusão de sangue pré-doado em cirurgia cardiaca com circulação extracorpórea. Rev. Bras. Cir. Cardiovasc., 4(2): 143-150, 1989.

rante o periodo de duração deste estudo, $38 \%$ dos pacientes operados foram submetidos a AD e AT, percentual que dificilmente pode ser considerado baixo.

As proteínas plasmáticas não são significantemente alteradas pela $A D$ e a volemia é recomposta em 12 horas $^{7}$. O fator pré-operatório de maior influência na previsão da necessidade transfusional em cirurgia coronária é o volume de células vermelhas ${ }^{4}$, e o fator limitante da recuperação deste índice é a reserva de ferro corpo$\mathrm{ral}^{8}$. NEWMAN et alii ${ }^{20}$ utilizando administração de ferro parenteral, descreveu autodoação de até quatro unidades em sete dias. Nesta série, optamos pelo uso de sulfato ferroso oral, mais facilmente tolerado.

A queda de 2,4 pontos percentuais entre o hematócrito pré-autodoação e o pré-operatório no Grupo GAD foi semelhante à descrita em outras séries ${ }^{6-8,16 \cdot 18,20}$. Embora, o hematócrito pré-operatório e durante CEC tenha sido mais baixo que no Grupo GC ( $<<0,05)$, este resultado carece de significado prático; o hematócrito pré-operatório do Grupo GAD estava dentro dos limites da normalidade e a anemia normovolêmica é bem tolerada durante a CEC, durante a retirada de CEC, e no pós-operatório ${ }^{23}$. Os dois grupos apresentaram hematócritos de chegada a UTI e alta similares, em torno dos $30 \%$, como descrito por outros autores ${ }^{6}, 7,16-18,29$.

A segurança com que a $A D$ pode ser realizada em cardiacos, inclusive em coronários, foi demonstrada por COVE et alii ${ }^{6}$ e LOVE et alii ${ }^{16}$; em 342 pacientes potencialmente de "alto risco", incluindo insuficiência cardiaca severa, insuficiência coronária, idosos, mulheres grávidas, crianças, a incidência de complicação de $A D$ não foi diferente da encontrada em doadores voluntários "normais" ${ }^{18}$. Em nosso estudo, tivemos um episódio de hipotensāo leve, assintomática, sem manifestação clínica ou eletrocardiográfica e rapidamente reversível, numa incidência de $2,4 \%$, comparável à descrita por MANN et alii ${ }^{18}$.

Os custos de uma unidade pré-doada foram estimados por LOVE et alii ${ }^{16}$ em US\$ 166,50 , igual ao de uma unidade homóloga; nāo podemos avaliar os custos em nosso país, por não dispormos de traballho neste sentido na literatura brasileira.
A AD e AT tem reduzido o volume de sangue homó$\operatorname{logo}$, sendo 37 a $68 \%$ do volume utilizado proveniente de sangue autógeno ${ }^{6,7},{ }^{16}$; na nossa série, que inclui sangue e hemoderivados homólogos na computação dos dados, $64 \%$ do volume utilizado foi proveniente de unidades autógenas. Consideramos que a computação de todos os hemoderivados homólogos é mais apropriada, desde que a morbidade não é exclusividade do sangue total.

A autodoação intra-operatória reduz o número de unidades homólogas usadas em cirurgia cardiaca ${ }^{9}$. ${ }^{10}$; redução ainda maior é obtida com a associação a autotransfusāo de plasma autógeno rico em plaquetas coletado inta-operatoriamente, utilizando-se 6,32 unidades homólogas por paciente ${ }^{16}$. A diferença entre o número médio de unidades homólogas utilizadas pelo Grupo GC (6,31/paciente) e pelo grupo GAD (1,95/paciente) demonstra uma utilização $223 \%$ acima pelo CG, comparando-se favoravelmente com o número obtido por GIORDANO et alii ${ }^{10}$.

O número de pacientes expostos a hemoderivados homólogos é reduzido por AD e AT ${ }^{6},{ }^{16}$. LOVE et alii ${ }^{16}$ relataram que apenas $32 \%$ dos pacientes com $A D$ e AT necessitaram sangue homólogo; o percentual similar obtido pelo nossso Grupo GAD, em que $23,8 \%$ dos pacientes necessitaram de SDH, inclui todos os hemoderivados.

Nossos resultados indicam que $\mathrm{AD}$ e $\mathrm{AT}$ são eficazes em reduzir o volume médio de $\mathrm{SDH}$ necessários em cirurgia cardiaca com CEC, diminuindo a exposição a unidades homólogas e minimizando o número de pacientes expostos. A análise do Grupo GAD I com o GAD II, embora renha demonstrado resultados sempre favoráveis ao GAD II, estes resultados não atingem significado estatístico, provavelmente devido ao pequeno número de pacientes em estudo.

Acreditamos que a implantação de um programa de $A D$ não apresenta grandes dificuldades. $A$ aceitação de $A D$ e $A T$ favorável pelos pacientes, a alta aplicabilidade destas técnicas e sua segurança, aliadas aos benefícios aqui demonstrados, sugerem que devem ser incentivados em cirurgia cardíaca eletiva. 
COSTA, M. G.; VASCONCELOS, F.; PEREIRA, R.; SILVEIRA, C. A.; GASPAR, E.; GODOY, G.; GARRET, M.; SOUZA, M. L.; LEĀO, A. C. - Autodoaçāo e autotransfusāo de sangue pré-doado em cirurgia cardiaca com circulação extracorpórea. Rev. Bras. Cir. Cardiovasc., 4(2): 143-150, 1989.

\section{RBCCV 44205-85}

COSTA, M G.; VASCONCELOS, F.; PEREIRA, R.; SILVEIRA, C. A.; GASPAR, E.; GODOY, G.; GARRET, M.; SOUZA, M. L.; LEĀO, A. C. - Autodonation and autotransfusion of pre-donated blood in cardiac surgery with cardiopulmonary bypass. Rev. Bras. Cir. Cardiovasc., 4(2): 143-150, 1989.

ABSTRACT: This is a prospective study performed from August 1987 to September 1988, including 80 adult patients who underwent elective cardiac surgery with cardiopulmonary bypass, for the purpose of assessing the efficacy of autodonation (AD) and autotransfusion (AT) in reducing the use of homologous blood and blood products (SDH). The Control Group (GC) did not undergo autodonation $(n=38)$. Pre-operative collection of flood was performed in $\mathbf{4 2}$ patients, forming the autodonation Group (GAD), beginning from 1 to 7 days pre-operatively (GAD I; $n=29$ ), and from 8 to 14 days pre-operatively (GAD II; $n=13$ ). Groups GC and GAD had very similiar "pre". "intra" and "post-operative" parameters. The results showed that the use of blood and blood products was similar in the various groups. Nevertheless, the number of patients exposed to SDH (GC $27(71 \%) \times$ GAD $10(23.8 \%)-p<0.001)$, the mean volume of SDH (GC 1241 $x$ GAD $412 \mathrm{ml}-\mathrm{p}<0.003$ ) and the mean number of Homologous Units used (GC $6.31 \times$ GAD 1.95 $-p<0.001$ ) demonstrated that $A D$ and AT were efficacious, reducing in $64 \%$ the mean volume of $S D H$, diminishing the exposure to Homologous Units and minimizing the number of patients exposed. The GAD II attained the best indexes, not reaching, however, statistical significance when compared to GAD I, possibility due to the small number of patients. We believe that AT \& AD should de encouraged in elective cardiac surgery. lation.

DESCRIPTORS: blood, autodonation; blood, autotransfusion; blood, conservation; extracorporeal circu-

\section{REFERÊNCIAS BIBLIOGRÁFICAS}

1 ALTER, M. J.; GERETY, F. J.: SMALLWOOD, L. A.; SAMPLINER, R. E.; TABOR, E.; DEINHARDT, F.; FORSNER, G.; MATANOSKI, G. M. - Sporadicnon-A, non-B hepatitis: frequency, epidemiology in an urban U.S. population. J. Infect.Dis., 145: 886-893, 1982.

2 BERMAN, M.; ALTER, H. J.; ISHAK, K. G.; PURCELL, R. H.; JONES, E. A. - The chronic sequelae of non-A, non-B hepatites. Ann. Intern. Med., 91: 1-6, 1979.

COSGROVE, D. M.; AMIOT, D. M.; MESERKO, J. J. An inproved technique for autotransfusion of shed mediastinal blood. Ann. Thorac. Surg., 40: 519-600, 1985.

4 COSGROVE, D. M.; LOOP, F. D.; LYTLE, B. W.; GILL, C. G.; GOLDING, L. R.; TAYLOR, P. C.; FORSYTHE, S. B. - Determinants of blood utilization during myocardial revascularization. Ann. Thorac. Surg., 40: 380-384, 1985.

5 COSGROVE, D. M.; THURER, R. L.; LYTLE, B. W.; GILL, C. G.; PETER, M.; LOOP, F. D. - Blood conservation during myocardial revascularization. Ann. Thorac. Surg., 28: 184-189, 1979.

6 COVE, H.; MATLOFF, J.; SACKS, H. J.; SHERBECOE, R.; GOLDFINGER, D. - Autologous blood transfusion in coronary artery bypass surgery. Transfusion, 16: 245-248, 1976.

7 Cuello, L.; VAzQuez, e.; Rios, R.; Rafucci, F. L. - Autologous blood transfusion in thoracic and cardiovascular surgery. Surgery, 62: 814-818, 1967.
8 FINCH, F.; HASKINS, D.; FINCH, C. A. - Iron metabolism. Hematopoiesis following phlebotomy: iron as a limiting factor. J. Clin. Invest., 29: 1078-1085, 1950.

9 GIORDANO, G. F.; GODMAN, D. S.; MAMMANA, R. B.; MARCO, J. D.; NESTOR, J. D.; RACZKOWSKI, A. R.; RIVERS, S. L.; SANDERSON, R. G. STRUG, B. S.; SANDLER, S. G. - Intraoperative autotranfusion in cardaic operations: effect on intraoperative and postoperative transfusion requirements. J. Thorac. Cardiovasc. Surg., 96: 382-386, 1988.

10 GIORDANO, G. F.; RIVERS, S. L.; CHUNG, G K. T.; MAMMANA, R. B.; MARCO, J. D.; RACZKOWSKI, A. R.; SABBAGH, A.; SANDERSON, R. G.; STRUG, B. S. - Autologous platelet-rich plasma in cardiac surgery: effect on intraoperative and postoperative transfusion requirements. Ann. Thorac. Surg., 46: 416-419, 1988.

11 HALLOWELL, P.; BLAND, J. H. L.; BUCKLEY, M. J.; LOWENSTEIN, E. - Transfusion of fresh autologous blood in open-heart surgery: a method for reducing bank blood requirements. J. Thorac. Cardiovasc. Surg., 64: 941-948, 1972.

12 HONIG, C. L. \& BOVE, J. R. - Transfusion associated fatalities: Review of Bureau of Biologics Reports 1976-1978. Transfusion, 20: 653-661, 1980.

13 JOHNSON, R. G.; ROSENKRANTZ, K. R.; PRESTON, R. A.; HOPKINS, C.; DAGGENT, W. M. - The efficacy of postoperative autotransfusion in patients undergoing cardiac operations. Ann. Thorac. Surg., 36:173-179, 1983. 
COSTA, M. G.; VASCONCELOS, F.; PEREIRA, R.; SILVEIRA, C. A.; GASPAR, E.; GODOY, G.; GARRET, M.; SOUZA, M. L.; LEĀO, A. C. - Autodoação e autotransfusão de sangue pré-doado em cirurgia cardíaca com circulação extracorpórea. Rev. Bras. Cir. Cardiovasc., 4(2): 143-150, 1989.

14 LINKER, C. \& WALLERSTEIN, R. O. - Blood transfusion. In: SCHOROEDER, S. A.; KRUPP, M. A.; THERNEY Jr., E. M. - Current medical diagnosis and treatment. 27. ed. Connecticut, Appleton \& Lange, 1988. p. 338-341.

15 LOOP, F. D.; WILCOX, B. R.; CUNNINGHAM Jr., J. M.; FOSBURG, R. G.; GEHA, A. S.; LAKS, H.; MARK, J. B. D.; BADHWAR, K. - Thoracic surgery manpower: the fourth manpower study of thoracic surgery: 1985. Report of the Ad Hoc Committee on Manpower of the American Association for Thoracic Surgry and the Society of Thoracic Surgeons. Ann. Thorac. Surg., 44: 450-461, 1987.

16 LOVE, T. R.; HENDREN, W. G.; O'KEEFE, D. D.; DAGGETT, W. M. - Transfusion of predonated autologous blood in elective cardiac surgery. Ann. Thorac. Surg., 43: $508-512,1987$.

17 LUBIN, J.; GREENBERG, J. J.; YAHR, W. Z; HAYNES, J. I.; PAUL, E. - The use of autologous blood in openheart surgery. Transfusion, 14:602-607, 1974.

18 MANN, M.; SACKS, H. J.; GOLDFINGER, D. - Safery of autologous blood donation prior to elective surgery for a variety of potentially "high-risk" patients. Transfusion, 23: 229-232, 1983.

19 MORAN, J. M.; BABKA, R.; SILBERMAN, S.; RICE, P. L.; PIFARRÉ, R.; SULLIVAN, H. J.; MONTOYA, A. Immediate centrifugation of oxygenator contents after cardiopulmonary bypass: role in maximum blood conservation. J. Thorac. Cardiovasc. Surg., 76:510-517. 1978.
20 NEWMANN, M. M.; HAMSTRA, R.; BLOCK, M. - Use of banked autologous blood in elective surgery. JAMA 218: 861-863, 1971

21 ROCHE, J. K. \& STENGLE, J. M. - Open-heart surgery and the demand for blood. JAMA, 225: 1516-1521, 1973.

SCHWARTZ, S. I. - Hemostasis, surgical bleeding and transfusion. In: SCHWARTZ, S. I.; SIRES, G. T.; SPENCER, F. C.; STORER, E. H. Principles of surgery. 3. ed. New York, McGraw Hill Inc., 1979. p. 129-130.

23 TECTOR, A. J.; GABRIEL, R. P.; MATEICKA, W. E.; OPARAH, S. S.; FLEMMA, R. J.; LEPLEY Jr., D. - Reduction of blood usage in open heart surgery. Chest, 70: $454-457,1976$

24 WILSON, J. D. \& TASWELL, H. F. - Autotransfusion: historical review and preliminary report on a new method. Mayo Clin. Proc., 43: 26-35, 1968.

25 YEH Jr., T.; SHELTON, L.; YEH, T. J. - Blood loss and bank blood requirement in coronary bypass surgery. Ann. Thorac. Surg., 26: 11-16, 1978.

26 AUHDI, N.; Mc COLLOUGH, B.; CAREY, J.; GREER, A. - Double-helical reservoir heart-lung machine designed for hypothermic perfusion primed with $5 \%$ glucose in water inducing hemodilution. Arch. Surg., 82: 320-325, 1961

AGRADECIMENTO: Agradecemos a Maria Valéria Gesteira Costa e Ana Elisa Latache a ajuda valiosa no preparo deste manuscrito. 